\title{
Cerebral Small Vessel Disease and Chronic Kidney Disease
}

\author{
Kazunori Toyoda \\ Department of Cerebrovascular Medicine, National Cerebral and Cardiovascular Center, Suita, Osaka, Japan
}

Chronic kidney disease, defined by a decreased glomerular filtration rate or albuminuria, is recognized as a major global health burden, mainly because it is an established risk factor for cardiovascular and cerebrovascular diseases. The magnitude of the effect of chronic kidney disease on incident stroke seems to be higher in persons of Asian ethnicity. Since the kidney and brain share unique susceptibilities to vascular injury due to similar anatomical and functional features of small artery diseases, kidney impairment can be predictive of the presence and severity of cerebral small vessel diseases. Chronic kidney disease has been reported to be associated with silent brain infarcts, cerebral white matter lesions, and cerebral microbleeds, independently of vascular risk factors. In addition, chronic kidney disease affects cognitive function, partly via the high prevalence of cerebral small vessel diseases. Retinal artery disease also has an independent relationship with chronic kidney disease and cognitive impairment. Stroke experts are no longer allowed to be ignorant of chronic kidney disease. Close liaison between neurologists and nephrologists can improve the management of cerebral small vessel diseases in kidney patients.

Keywords Acute stroke; Asian; Cognitive impairment; Dementia; Lacunar infarction; Retinopathy

\author{
Correspondence: Kazunori Toyoda \\ Department of Cerebrovascular \\ Medicine, National Cerebral and \\ Cardiovascular Center, \\ 5-7-1 Fujishiro-dai, Suita, Osaka \\ 565-8565, Japan \\ Tel: +81-6-6833-5012 \\ Fax: +81-6-6835-5267 \\ E-mail: toyoda@ncvc.go.jp
}

Received: August 25, 2014 Revised: November 24, 2014 Accepted: November 24, 2014

The author has no financial conflicts of interest.

\section{Introduction}

Chronic kidney disease (CKD) is defined by a decreased estimated glomerular filtration rate (eGFR) below $60 \mathrm{~mL} / \mathrm{min} / 1.73$ $\mathrm{m}^{2}$ or albuminuria as a marker of an increased glomerular permeability. ${ }^{1}$ Beyond the original meaning of $\mathrm{CKD}$ as a high-risk state for future dialysis, $\mathrm{CKD}$ is now recognized as a significant and rapidly growing global health burden, mainly because it is an established risk factor for cardiovascular diseases, including stroke. $^{2,3}$ The impact of CKD on incident stroke differs among regions and races and is higher in Asian people. ${ }^{4}$ The glomerular afferent arterioles of the juxtamedullary nephrons and the cerebral small vessels have anatomical and functional features in common. ${ }^{5}$ Thus, cerebral small vessel disease (SVD) and CKD seem to have a strong relationship. In this paper, the association between $\mathrm{CKD}$ and overall stroke is reviewed first, and then the special contribution of CKD to symptomatic and subclinical cerebral SVDs is discussed.

\section{Chronic kidney disease is a risk factor for stroke}

$\mathrm{CKD}$ is prevalent in stroke patients. Figure 1 shows the prevalence of eGFR below $60 \mathrm{~mL} / \mathrm{min} / 1.73 \mathrm{~m}^{2}$ in both the general population and acute stroke patients. ${ }^{3}$ For acute stroke patients, eGFR was assessed using creatinine levels during acute stroke and was accordingly affected by acute stroke damage. The prevalence varies from $20 \%$ to $35 \%$ in patients with acute ischemic stroke ${ }^{2,6-10}$ and from $20 \%$ to $46 \%$ in patients with acute intracerebral hemorrhage (ICH). ${ }^{2,6,11,12}$ This prevalence was found to be 


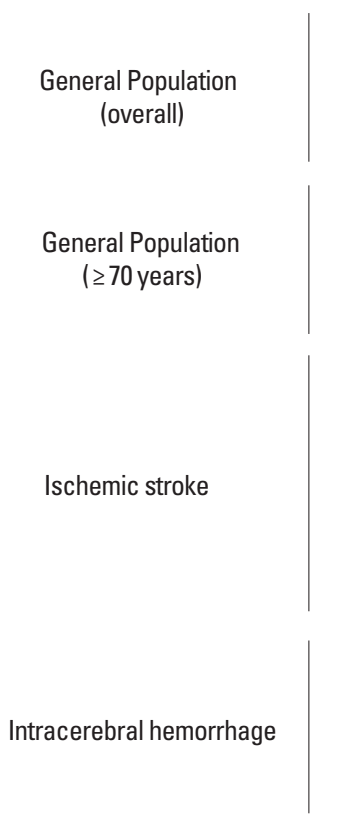

NHANES'99-'04 (US) [13]

HUNT II (Norway) [14]

Imai, et al. (Japan) [15]

NHANES'99-'04 (US)

HUNT II (Norway)

Imai, et al. (Japan)

Yahalom, et al. [6]

Ovbiagele, et al. [7]

PRoFESS [8]

SAMURAI rt-PA Registry [9]

Fukuoka Stroke Registry [10]

Toyoda [2]

Yahalom, et al. [6]

Molshatzki, et al. [11]

SAMURAI ICH Study [12]

Toyoda [2]

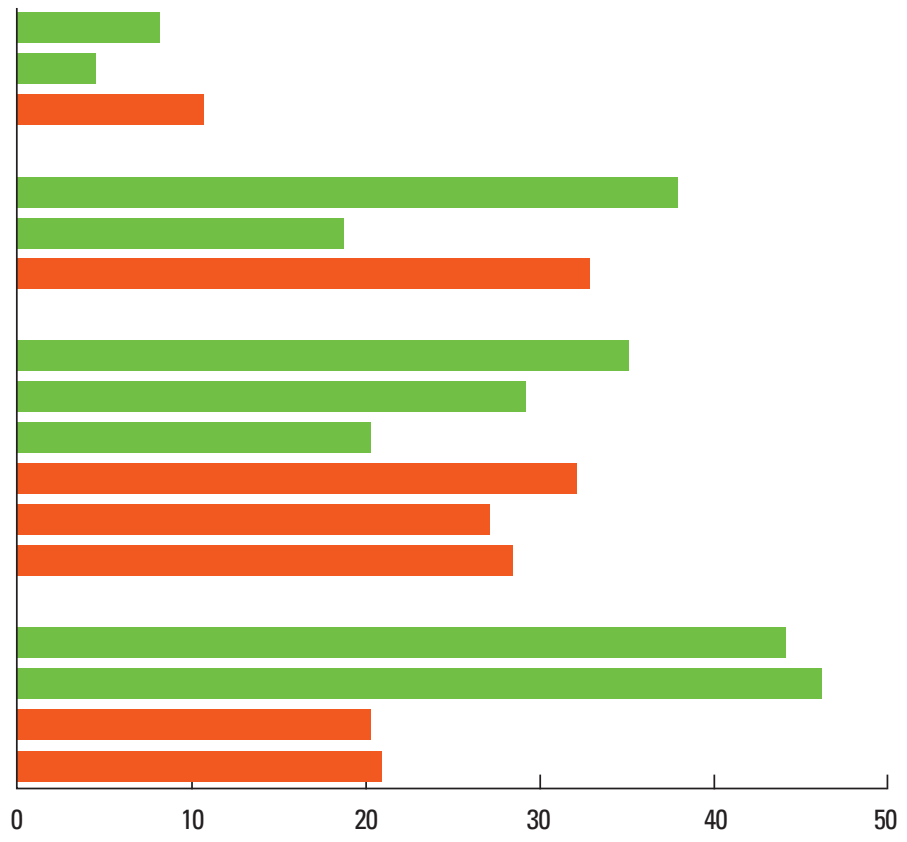

(\%)

Figure 1. The prevalence of eGFR $<60 \mathrm{~mL} / \mathrm{min} / 1.73 \mathrm{~m}^{2}$ in the general population and stroke patients.

Orange bars indicate data from Japanese subjects.

Mean age (years): [2] 70.1 (overall stroke), [7] 66, [8] 66.0, [9] 71.4, [10] 70.0, [11] 71.7, [12] 65.6, [13] 46.2, [14] 50.2. From reference 3 with permission.

higher than that in the general population $(4.4 \%$ to $11 \%)$ and similar to that in the elderly general population aged 70 years or older (19\% to $38 \%){ }^{13-15}$ Stroke occurs much more commonly in dialysis patients than in the general population or $\mathrm{CKD}$ patients without need for dialysis. In my previous studies in a kidney center in the Fukuoka metropolitan area in western Japan, the incidence of total stroke for patients receiving hemodialysis was 13 per 1,000 person-years, and that for patients receiving continuous ambulatory peritoneal dialysis was 15.7 per 1000 personyears. ${ }^{16,17}$

$\mathrm{CKD}$ is an established risk factor for stroke. A meta-analysis of 21 prospective cohort studies, involving 284,672 participants, showed that the risk of stroke increased by $43 \%$ [ $95 \%$ confidence interval (CI) $31 \%-57 \%$ ] in subjects with an eGFR $<60 \mathrm{~mL} / \mathrm{min} / 1.73 \mathrm{~m}^{2}{ }^{2}$ Lower eGFR was a risk factor for both ischemic and hemorrhagic stroke. In another meta-analysis of 10 prospective cohort studies involving 140,231 participants, participants with proteinuria had a $71 \%$ (95\% CI 39\%-110\%) greater risk of stroke than those without proteinuria. ${ }^{18}$

Racial difference was mentioned in these two meta-analyses., ${ }^{4,18}$ The impact of the decreased eGFR on stroke was higher in Asian populations [risk ratio (RR) $1.96,95 \% \mathrm{CI} 1.73$ to 2.23 ] than in non-Asians $(1.26,1.16-1.35, P<0.001)$ in the former one. ${ }^{4}$ In the latter one ${ }^{18}$ the impact of proteinuria was weaker in Asians than in non-Asians (RR 1.43, 95\% CI, 1.14-1.79 vs. 2.02, 1.54-2.65; $P$ for heterogeneity 0.07 ), and there was no evidence to support an ethnic difference in the magnitude of the association after adjusting for method of protein measurement. In the Reasons for Geographic and Racial Differences in Stroke (REGARDS) Study, involving 25,310 community-dwelling participants over 44 years old, a higher urinary albumin to creatinine ratio (ACR) was associated with stroke risk independently of eGFR and traditional risk factors among black participants, and the association was modest and not independent among white participants. ${ }^{19}$

\section{Chronic kidney disease affects stroke severity and outcomes}

In addition to the increase in the risk of incident stroke, CKD affects initial neurological severity and chronic outcomes of ischemic and hemorrhagic stroke. The Fukuoka Stroke Registry is a large multicenter registry for acute stroke patients in the Fukuoka metropolitan area. Of 3,778 patients with first-ever ischemic stroke from the registry, 1,320 (34.9\%) were diagnosed as having CKD. ${ }^{10}$ These CKD patients had a 49\% (95\% CI 17\%-89\%) greater risk of neurological deterioration during hospitalization, defined as a $\geq 2$-point increase in the National Institutes of Health Stroke Scale score; a 138\% (95\% CI 61\%-257\%) greater risk of in-hospital mortality; and a $25 \%$ (95\% CI 5\%-48\%) greater risk of a modified Rankin Scale (mRS) score $\geq 2$ at discharge than non-CKD patients, after adjustment for potential confounding factors, including initial stroke severity. From the Fu- 
kuoka Stroke Registry, a 73\% (95\% CI 3\%-190\%) greater risk of recurrence of noncardioembolic stroke in CKD patients was also reported. ${ }^{20}$ The China National Stroke Registry, involving 4,836 diabetic patients with acute stroke and transient ischemic attack (TIA) showed that eGFR $<45 \mathrm{~mL} / \mathrm{min} / 1.73 \mathrm{~m}^{2}$ was independently associated with risks of all-cause death, recurrent stroke, combined endpoint of stroke or death, and stroke disability in patients with overall stroke/TIA and those with ischemic stroke/TIA. ${ }^{21}$

We determined the safety and feasibility of systolic blood pressure reduction initiated within 3 hours from onset to $<160 \mathrm{mmHg}$ using intravenous nicardipine in 211 patients with acute spontaneous intracerebral hemorrhage (ICH) in the prospective, multicenter, observational Stroke Acute Management with Urgent Riskfactor Assessment and Improvement (SAMURAI)-ICH Study. ${ }^{22}$ In its subanalysis, eGFR $<60 \mathrm{~mL} / \mathrm{min} / 1.73 \mathrm{~m}^{2}$ was positively associated with mRS score 5-6 (OR 5.87, 95\% CI 1.87-19.34) and negatively associated with $\mathrm{mRS}$ score 0-2 (OR 0.21, 95\% CI 0.070.54 ) at 3 months, after adjustment for known prognostic predictors including the initial NIH Stroke Scale score and hematoma volume. ${ }^{12}$ In several other studies, the effects of CKD on neurological severity and outcomes of ischemic and hemorrhagic stroke were clarified. ${ }^{2,3}$

Resistance to and limitations of stroke therapies appear to partly cause the poorer stroke outcomes of CKD patients. CKD patients have both high thromboembolic risk and high bleeding risk, and it is often difficult to maintain the balance of the risks and benefits of antithrombotic therapy. There is conflicting evidence for the benefit of stroke prevention with warfarin in patients with advanced CKD and end-stage kidney disease; some supported a protective effect of warfarin from stroke ${ }^{23}$ and others denied it. ${ }^{24,25}$ Non-vitamin Kantagonist oral anticoagulants (NO$\mathrm{ACs}$ ) cannot be given to patients with advanced $\mathrm{CKD}^{26}$ In our multicenter SAMURAI rt-PA Registry, ${ }^{9}$ a decreased eGFR $<60$ $\mathrm{mL} / \mathrm{min} / 1.73 \mathrm{~m}^{2}$ on admission was associated with early symptomatic ICH [odds ratio (OR) 1.81, 95\% CI 1.16-2.84], mortality $(2.94,1.38-6.42)$, and $\mathrm{mRS}$ score $\geq 4$ at 3 months after intravenous thrombolysis using alteplase at $0.6 \mathrm{mg} / \mathrm{kg}$ for ischemic stroke patients (1.55, 1.01-2.38). A meta-analysis was performed based on three studies including the SAMURAI rt-PA Registry; it showed significant associations of reduced eGFR with early symptomatic ICH, mortality, and poor functional outcome. ${ }^{27}$

\section{Anatomical relationship between cerebral small vessel disease and kidney disease}

injury since the vasoregulation of the microvasculatures of the two organs are similar anatomically and functionally. ${ }^{5}$ Both the glomerular afferent arterioles of the juxtamedullary nephrons and the cerebral perforating arteries are small, short vessels directly arising from large high-pressure arteries. The arteries are accordingly exposed to a high pressure, and they have to maintain a strong vascular tone in order to provide a large pressure gradient in a short distance. Ito et al referred to these kinds of vessels as 'strain vessels' and listed coronary microcirculation and retinal arteries as other strain vessels. Hypertensive vascular damage occurs first and severely in such strain vessels. Since albuminuria reflects glomerular damage distal to the juxtamedullary afferent arterioles, albuminuria may also be an early sign of cerebral small vessel damage.

\section{Chronic kidney disease and silent lacunar infarction}

Although epidemiological studies did not mention the contribution of CKD to subtypes of incidental stroke, a relationship between CKD and silent brain infarction (SBI), mainly lacunar infarction, has been reported both in cohort studies and in hospital-based studies. An inverse linear association was observed between 1 /cystatin $\mathrm{C}$, a marker of kidney function, and the prevalence of SBIs after multivariate adjustment [OR 1.20, 95\% CI 1.09-1.32 per 1-standard deviation (SD) decrease] in older adults from the Cardiovascular Health Study found. ${ }^{28}$ In the Rotterdam Scan Study, a decreased eGFR tended to be associated with a higher prevalence of SBIs after age- and sex-adjustment (OR 1.11, 95\% CI 0.81-1.51 per 1-SD decrease). ${ }^{29}$ In a cross-sectional study involving 625 community-based Japanese elderly participants, $\mathrm{CKD}$ was independently associated with lacunar infarction after multivariate adjustment (OR 1.86, 95\% CI 1.28-2.72) ${ }^{30} \mathrm{CKD}$ remained an independent risk factor by excluding diabetic subjects or subjects with stage 2 hypertension.

Two hospital-based studies from Japan are also relevant. In the first study, silent lacunar infarction was identified on magnetic resonance imaging (MRI) in $25 \%$ of patients with a creatinine clearance $>40 \mathrm{~mL} / \mathrm{min} / 1.73 \mathrm{~m}^{2}$ and in $85 \%$ of patients with creatinine clearance $<40 \mathrm{~mL} / \mathrm{min} / 1.73 \mathrm{~m}^{2}$; the strongest contributing factor for lacunar infarction on multivariate analysis was decreased creatinine clearance. ${ }^{31}$ In another one, SBI was identified on MRI in 108 of 324 (31.8\%) predialysis CKD patients. ${ }^{32}$ As the CKD stage advanced, SBI was more commonly identified $(P<0.0001)$. A decreased eGFR was independently associated with SBI.

The kidney and brain share unique susceptibilities to vascular 


\section{Chronic kidney disease and white matter lesions}

Like SBIs, white matter lesions have often been studied to clarify their association with CKD. The Northern Manhattan Study, involving 615 stroke-free participants, showed that eGFR of $15-60 \mathrm{~mL} / \mathrm{min} / 1.73 \mathrm{~m}^{2}$ was significantly associated with an increased log-transformed volume of white matter lesions $(\beta$ 0.322 [95\% CI 0.080-0.564]) after adjusting for cardiovascular risk factors. ${ }^{33}$ With 484 elderly inhabitants (60-90 years of age) in the Rotterdam Scan Study involving 484 participants 60 years old or older, the volume of deep white matter was significantly decreased, and the volume of white matter lesions was increased as eGFR decreased after multivariate adjustment. ${ }^{29}$

\section{Chronic kidney disease and cerebral microbleeds}

Cerebral microbleeds (CMBs), another typical cerebral SVD, were identified in 35 of 162 patients $(25.6 \%)$ with $\mathrm{CKD}$ stages 1 to 5 not on dialysis. ${ }^{34}$ In this cohort, eGFR was inversely associated with the presence of CMBs independent of sex, age, and diastolic blood pressure (OR 0.956, 95\% CI 0.926-0.988 per 1 $\mathrm{mL} / \mathrm{min}$ increase). In 236 consecutive inpatients with acute ischemic stroke or TIA, proteinuria had strong independent associations with both the presence (OR 2.33, 95\% CI 1.19-4.95) and number $(2.23,1.10-4.53)$ of CMBs. ${ }^{35}$ In 144 black patients with recently developed ICH from the DiffErenCes in the Imaging of Primary Hemorrhage based on Ethnicity or Race (DECIPHER) study, CKD was also associated with both the presence (OR 3.44, 95\% CI, 1.64-7.24) and number (2.46, 1.11-5.42) of CMBs, but this was not true in non-Hispanic white patients. ${ }^{36}$

\section{Chronic kidney disease affects cognitive function}

Dementia is one of the greatest global health burdens along with CKD. Patients at all stages of CKD have a higher risk of developing cognitive impairment than the general population. ${ }^{37}$ Its prevalence in patients on hemodialysis has been estimated at $30 \%$ to $60 \%$, at least twice the values observed in age-matched controls, with a similar prevalence for peritoneal dialysis patients. ${ }^{38}$ Additionally, cognitive impairment is more common in milder CKD than in the general population. In 23,405 communitydwelling participants $>44$ years old from the REGARDS Study, eGFR $<60 \mathrm{~mL} / \mathrm{min} / 1.73 \mathrm{~m}^{2}$ (OR 1.23, 95\% CI 1.06-1.43), as well as each $10 \mathrm{~mL} / \mathrm{min} / 1.73 \mathrm{~m}^{2}$ decrease of eGFR (OR 1.11, 95\% CI 1.04-1.19), was independently associated with an in- creased risk of cognitive impairment after adjustment for vascular risk factors and others. ${ }^{39}$

There seems to be a few factors to cause cognitive dysfunction in CKD patients. First, direct neuronal toxicity by uremic toxins seems to be another cause of cognitive impairment in CKD patients. ${ }^{40}$ Second, conventional vascular risk factors that are common to CKD patients, such as chronic hypertension, diabetes, smoking, and obesity, can be risk factors for Alzheimer's disease. ${ }^{41}$ In a recent report from the ongoing Osaka Followup Study for Carotid Atherosclerosis Part 2 (OSACA2) study involving 600 participants with vascular risk factors, 50 subjects developed dementia during the median 7.5-year follow-up. It was found that eGFR $<60 \mathrm{~mL} / \mathrm{min} / 1.73 \mathrm{~m}^{2}$ at entry was independently related to the risk of all-cause dementia even after adjusting for brain atrophy and cerebral small vessel diseases on MRI (RR 1.96, 95\% CI 1.08-3.58) ${ }^{42}$ As the third factor, several previous studies often explain the potential association between $\mathrm{CKD}$ and cognitive impairment via the high prevalence of SBI, white matter lesions, and CMBs. ${ }^{43}$

CKD also affects longitudinal cognitive change. In the 7,839 participants of the 3C Study, eGFR decline for more than 4 years, but not the baseline eGFR, was associated with decline in global cognition assessed by the Mini-Mental State Examination. ${ }^{4}$

\section{Chronic kidney disease is associated with retinal artery disease}

Retinopathy and nephropathy are known to be major diabetic microvascular complications. In addition, the glomerular afferent arterioles and retinal arteries are both strain arteries.' Thus, an association between $\mathrm{CKD}$ and retinal artery disease has been reported. In the Singapore Prospective Study Program, involving 261 participants with eGFR $<60 \mathrm{~mL} / \mathrm{min} / 1.73 \mathrm{~m}^{2}$ and 651 controls, the lowest and highest quintiles of the retinal fractal dimension were associated with increased prevalence of $\mathrm{CKD}$ after multivariate adjustment, even in participants without diabetes. ${ }^{45}$ This result indicates that kidney dysfunction affects deviations from optimal microvascular architecture independently of diabetes. In the Chronic Renal Insufficiency Cohort (CRIC) study involving 1904 participants, patients with eGFR of 30-39 $\mathrm{mL} / \mathrm{min} / 1.73 \mathrm{~m}^{2}$ had a $98 \%$ (95\% CI 38\%-183\%) greater risk, and those with eGFR $<30 \mathrm{~mL} / \mathrm{min} / 1.73 \mathrm{~m}^{2}$ had a $199 \%$ (95\% CI 105\%-306\%) greater risk of retinopathy after multivariate adjustment than those with eGFR $\geq 50 \mathrm{~mL} / \mathrm{min} / 1.73 \mathrm{~m}^{2} .{ }^{46}$

$\mathrm{CKD}$, retinopathy, and cognitive impairment share the common vascular pathology of SVDs. In 2,211 participants undergoing retinal photography in the Cardiovascular Health Study, retinopathy was associated with poorer scores on the Digit Symbol 
Substitution Test. ${ }^{47}$ In the 14-year follow-up study of 803 participants from the Atherosclerosis Risk in the Community (ARIC) cohort, subjects with retinopathy showed more rapid decline of the scores of the Word Fluency test and the Digit Symbol Substitution test than those without retinopathy. ${ }^{48}$ In the CRIC study involving 588 participants, retinopathy was independently associated with cognitive impairment on the Trail-Making Test, Forms A (OR 3.4, 95\% CI 2.0-6.0), Forms B (3.0, 1.8-4.9), and the Boston Naming Test $(2.1,1.2-3.4){ }^{49}$ The relationship between retinopathy and CKD seems to be stronger in patients with type 2 diabetes. The CKD stage was significantly and independently associated with decreased retinal blood flow measured by a Doppler velocimetry system in 169 Japanese patients with type 2 diabetes. ${ }^{50}$

\section{Conclusion}

The strong associations of CKD with cerebrovascular diseases, especially with cerebral SVDs, including retinal artery disease, have been reviewed. A deep understanding of the cerebrorenal interaction is important to minimize the burden of cerebrovascular disease and cognitive impairment in CKD patients. ${ }^{51}$ Close liaison between neurologists and nephrologists can improve the management of SVDs in CKD.

\section{References}

1. KDIGO Work Group. KDIGO 2012 clinical practice guideline for the evaluation and management of chronic kidney disease. 1: definition, and classification of CKD. Kidney Int Suppl 2013; 3: 19-62.

2. Toyoda K, ed. Brain, Stroke and Kidney. Basel: Karger, 2013

3. Toyoda K, Ninomiya T. Stroke and cerebrovascular diseases in patients with chronic kidney disease. Lancet Neurol 2014;13: 823-833

4. Lee M, Saver JL, Chang KH, Liao HW, Chang SC, Ovbiagele B. Low glomerular filtration rate and risk of stroke: meta-analysis. BMJ 2010;341:c4249.

5. Ito S, Nagasawa T, Abe M, Mori T. Strain vessel hypothesis: a viewpoint for linkage of albuminuria and cerebro-cardiovascular risk. Hypertens Res 2009;32:115-121.

6. Yahalom G, Schwartz R, Schwammenthal Y, Merzeliak O, Toashi $\mathrm{M}$, Orion $\mathrm{D}$, et al. Chronic kidney disease and clinical outcome in patients with acute stroke. Stroke 2009;40:1296-1303.

7. Ovbiagele B, Sanossian N, Liebeskind DS, Kim D, Ali LK, Pineda $S$, et al. Indices of kidney dysfunction and discharge outcomes in hospitalized stroke patients without known renal disease. Cerebrovasc Dis 2009; 28:582-588.
8. Ovbiagele B, Bath PM, Cotton D, Sha N, Diener HC; PRoFESS Investigators. Low glomerular filtration rate, recurrent stroke risk, and effect of renin-angiotensin system modulation. Stroke 2013;44:3223-3225.

9. Naganuma M, Koga M, Shiokawa Y, Nakagawara J, Furui E, Kimura $\mathrm{K}$, et al. Reduced estimated glomerular filtration rate is associated with stroke outcomes after intravenous rt-PA: the Stroke Acute Management with Urgent Risk-factor Assessment and Improvement (SAMURAI) rt-PA registry. Cerebrovasc Dis 2011;31:123-129.

10. Kumai Y, Kamouchi M, Hata J, Ago T, Kitayama J, Nakane H, et al. Proteinuria and clinical outcomes after ischemic stroke. Neurology 2012;78:1909-1915.

11. Molshatzki N, Orion D, Tsabari R, Schwammenthal Y, Merzeliak O, Toashi M, et al. Chronic kidney disease in patients with acute intracerebral hemorrhage: association with large hematoma volume and poor outcome. Cerebrovascular Dis 2011;31: 271-277.

12. Miyagi T, Koga M, Yamagami H, Okuda S, Okada Y, Kimura $\mathrm{K}$, et al. Reduced estimated glomerular filtration rate affects outcomes 3 months after intracerebral hemorrhage: the Stroke Acute Management with Urgent Risk-factor Assessment and Improvement-Intracerebral Hemorrhage study. J Stroke Cerebrovasc Dis 2014 Nov 6 [Epub ahead of print]

13. Coresh J, Selvin E, Stevens LA, Manzi J, KusekJW, Eggers P, et al. Prevalence of chronic kidney disease in the United States. JAMA 2007;298:2038-2047.

14. Hallan SI, Coresh J, Astor BC, Asberg A, Powe NR, Romundstad $S$, et al. International comparison of the relationship of chronic kidney disease prevalence and ESRD risk. J Am Soc Nephrol 2006; 17:2275-2284.

15. Imai E, Horio M, Watanabe T, Iseki K, Yamagata K, Hara S, et al. Prevalence of chronic kidney disease in the Japanese general population. Clin Exp Nephrol 2009; 13:621-630.

16. Toyoda K, Fujii K, Ando T, Kumai Y, Ibayashi S, Iida M. Incidence, etiology, and outcome of stroke in patients on continuous ambulatory peritoneal dialysis. Cerebrovasc Dis 2004; 17 : 98-105.

17. Toyoda K, Fujii K, Fujimi S, Kumai Y, Tsuchimochi H, Ibayashi $S$, et al. Stroke in patients on maintenance hemodialysis: a 22year single-center study. Am J Kidney Dis 2005;45:1058-1066.

18. Ninomiya T, Perkovic V, Verdon C, Barzi F, Cass A, Gallagher $\mathrm{M}$, et al. Proteinuria and stroke: a meta-analysis of cohort studies. Am J Kidney Dis 2009;53:417-425.

19. Gutiérrez OM, Judd SE, Muntner P, Rizk DV, McClellan WM, Safford MM, et al. Racial differences in albuminuria, kidney function, and risk of stroke. Neurology 2012;79:1686-1692.

20. Kuwashiro T, Sugimori H, Ago T, Kamouchi M, Kitazono T; 
FSR Investigators. Risk factors predisposing to stroke recurrence within one year of non-cardioembolic stroke onset: the Fukuoka Stroke Registry. Cerebrovasc Dis 2012;33:141-149.

21. Luo Y, Wang X, Wang Y, Wang C, Wang H, Wang D, et al. Association of glomerular filtration rate with outcomes of acute stroke in type 2 diabetic patients: results from the China National Stroke Registry. Diabetes Care 2014;37:173-179.

22. Koga M, Toyoda K, Yamagami H, Okuda S, Okada Y, Kimura $\mathrm{K}$, et al. Systolic blood pressure lowering to $160 \mathrm{mmHg}$ or less using nicardipine in acute intracerebral hemorrhage: a prospective, multicenter, observational study (the Stroke Acute Management with Urgent Risk-factor Assessment and Improvement-Intracerebral Hemorrhage study). J Hypertens 2012;30: 2357-2364.

23. Olesen JB, Lip GY, Kamper AL, Hommel K, Køber L, Lane DA, et al. Stroke and bleeding in atrial fibrillation with chronic kidney disease. N Engl J Med 2012;367:625-635.

24. Yang F, Chou D, Schweitzer P, Hanon S. Warfarin in haemodialysis patients with atrial fibrillation: what benefit? Europace 2010;12:1666-1672.

25. Chan KE, Lazarus JM, Thadhani R, Hakim RM. Anticoagulant and antiplatelet usage associates with mortality among hemodialysis patients. J Am Soc Nephrol 2009;20:872-881.

26. Ahmad Y, Lip GY. Preventing stroke and systemic embolism in renal patients with atrial fibrillation: focus on anticoagulation. Contrib Nephrol 2013;179:81-91.

27. Hirano T. Thrombolysis and hyperacute reperfusion therapy for stroke in renal patients. Contrib Nephrol 2013;179:110-118.

28. Seliger SL, Longstreth WT Jr, Katz R, Manolio T, Fried LF, Shlipak M, et al. Cystatin C and subclinical brain infarction. J Am Soc Nephrol 2005; 16:3721-3727.

29. Ikram MA, Vernooij MW, Hofman A, Niessen WJ, van der Lugt A, Breteler MM. Kidney function is related to cerebral small vessel disease. Stroke 2008;39:55-61.

30. Wada M, Nagasawa H, Iseki C, Takahashi Y, Sato H, Arawaka S, et al. Cerebral small vessel disease and chronic kidney disease (CKD): results of a cross-sectional study in community-based Japanese elderly. J Neurol Sci 2008;272:36-42.

31. Kobayashi S, Ikeda T, Moriya H, Ohtake T, Kumagai H. Asymptomatic cerebral lacunae in patients with chronic kidney disease. Am J Kidney Dis 2004;44:35-41.

32. Shima H, Ishimura E, Naganuma T, Ichii M, Yamasaki T, Mori $\mathrm{K}$, et al. Decreased kidney function is a significant factor associated with silent cerebral infarction and periventricular hyperintensities. Kidney Blood Press Res 2011;34:430-438

33. Khatri M, Wright CB, Nickolas TL, Yoshita M, Paik MC, Kranwinkel G, et al. Chronic kidney disease is associated with white matter hyperintensity volume: the Northern Manhattan Study
(NOMAS). Stroke 2007;38:3121-3126.

34. Shima H, Ishimura E, Naganuma T, Yamazaki T, Kobayashi I, Shidara K, et al. Cerebral microbleeds in predialysis patients with chronic kidney disease. Nephrol Dial Transplant 2010;25: 1554-1559.

35. Ovbiagele B, Liebeskind DS, Pineda S, Saver JL. Strong independent correlation of proteinuria with cerebral microbleeds in patients with stroke and transient ischemic attack. Arch Neurol 2010;67:45-50.

36. Ovbiagele B, Wing JJ, Menon RS, Burgess RE, Gibbons MC, Sobotka I, et al. Association of chronic kidney disease with cerebral microbleeds in patients with primary intracerebral hemorrhage. Stroke 2013;44:2409-2413.

37. Bugnicourt JM, Godefroy O, Chillon JM, Choukroun G, Massy ZA. Cognitive disorders and dementia in CKD: the neglected kidney-brain axis. J Am Soc Nephrol 2013;24:353-363.

38. Radić J, Ljutić D, Radić M, Kovaĉić V, Sain M, Curković KD. The possible impact of dialysis modality on cognitive function in chronic dialysis patients. Neth J Med 2010;68:153-157.

39. Kurella Tamura M, Wadley V, Yaffe K, McClure LA, Howard G, Go R, et al. Kidney function and cognitive impairment in US adults: the Reasons for Geographic and Racial Differences in Stroke (REGARDS) Study. Am J Kidney Dis 2008:52;227-234.

40. Seifter JL, Samuels MA. Uremic encephalopathy and other brain disorders associated with renal failure. Semin Neurol 2011;31: 139-143.

41. Daviglus ML, Bell CC, Berrettini W, Bowen PE, Connolly ES Jr, Cox NJ, et al. National Institutes of Health State-of-the-Science Conference statement: preventing alzheimer disease and cognitive decline. Ann Intern Med 2010;153:176-181.

42. Miwa K, Tanaka M, Okazaki S, Furukado S, Yagita Y, Sakaguchi $\mathrm{M}$, et al. Chronic kidney disease is associated with dementia independent of cerebral small-vessel disease. Neurology 2014;82: 1051-1057.

43. Elias MF, Dore GA, Davey A. Kidney disease and cognitive function. Contrib Nephrol 2013;179:42-57.

44. Helmer C, Stengel B, Metzger M, Froissart M, Massy ZA, Tzourio $\mathrm{C}$, et al. Chronic kidney disease, cognitive decline, and incident dementia: the 3C Study. Neurology 2011;77:2043-2051.

45. Sng CC, Sabanayagam C, Lamoureux EL, Liu E, Lim SC, Hamzah $\mathrm{H}$, et al. Fractal analysis of the retinal vasculature and chronic kidney disease. Nephrol Dial Transplant 2010;25:2252-2258.

46. Grunwald JE, Alexander J, Maguire M, Whittock R, Parker C, McWilliams K, et al. Prevalence of ocular fundus pathology in patients with chronic kidney disease. Clin J Am Soc Nephrol 2010;5:867-873.

47. Baker ML, Marino Larsen EK, Kuller LH, Klein R, Klein BE, Siscovick DS, et al. Retinal microvascular signs, cognitive func- 
tion, and dementia in older persons: the Cardiovascular Health Study. Stroke 2007;38:2041-2047.

48. Lesage SR, Mosley TH, Wong TY, Szklo M, Knopman D, Catellier DJ, et al. Retinal microvascular abnormalities and cognitive decline: the ARIC 14-year follow-up study. Neurology 2009; 73: 862-868.

49. Yaffe K, Ackerson L, Hoang TD, Go AS, Maguire MG, Ying GS, et al. Retinopathy and cognitive impairment in adults with CKD. Am J Kidney Dis 2013;61:219-227.

50. Nagaoka T, Yoshida A. Relationship between retinal blood flow and renal function in patients with type 2 diabetes and chronic kidney disease. Diabetes Care 2013;36:957-961.

51. Toyoda K. The cerebro-renal interaction in stroke neurology. Neurology 2012;78:1898-1899. 